\title{
AVALIAÇÃO DA RESPOSTA CLÍNICA DA ESTABILIZAÇÃO PEDICULAR, SEM FUSÃO, EM PACIENTES COM LOMBALGIA
}

\author{
CLINICAL OUTCOMES WITH NON-FUSION STABILIZATION IN LOW BACK PAIN \\ EVALUACIÓN DE LA RESPUESTA CLÍNICA DE LA ESTABILIZACIÓN PEDICULAR, SIN FUSIÓN, \\ EN PACIENTES CON LUMBALGIA
}

Fabrício Guedes Machado ${ }^{1}$, Marcos Masayuki Ishi ${ }^{1}$

\begin{abstract}
RESUMO
Objetivo: Avaliar o resultado clínico do tratamento cirúrgico da doença discal degenerativa (DDD) com o uso de sistemas de estabilização pedicular sem fusão em 22 pacientes na cidade de Aracaju/Sergipe. Métodos: Foram utilizadas escala visual analógica da dor (EVA) e qualidade de vida ( questionários Oswestry e Roland Moris) no pré-operatório e seis meses após o tratamento cirúrgico instituído. De acordo com as queixas clínicas e as alterações morfofisiológicas encontradas nos exames complementares de imagem, grau de acometimento articular (artrose facetária), doença discal em níveis adjacentes, microdiscectomias prévias, optou-se pela utilização de implantes que permitem graus de mobilidade variáveis. Resultados: Dos 22 pacientes incluídos no estudo, 16 eram do sexo masculino e seis do sexo feminino, com média de idade de 41,77 \pm 11,6 anos. Foi estabilizado um total de 43 níveis, sendo a instrumentação de L4 a S1 a mais frequente (12) e o sistema mais utilizado foi a placa Dinamika ${ }^{\circledR}$ (GMReis) para dois níveis (08), com fusão do nível inferior (estabilização

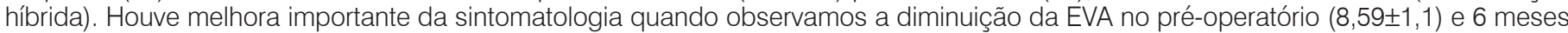
no pós-operatório $(2,41 \pm 1,8)$, com melhora na qualidade de vida nas escalas de Oswestry e Roland Moris (ODI pré: $55,18 \pm 15,5$ e ODI pós: 13,91 \pm 13,1 ; RM pré: 27,23 \pm 4,9 e RM pós: 41,68 \pm 4,8) respectivamente. Conclusão: Apesar de todos os pacientes incluídos no estudo terem obtido melhora expressiva tanto na qualidade de vida como na diminuição da sintomatologia, novos estudos biomecânicos devem ser realizados a fim de se padronizar uma abordagem eficiente no tratamento dessa patologia.
\end{abstract}

Descritores: Lombalgia, Doença discal degenerativa, Estabilização dinâmica.

\begin{abstract}
Objective: To evaluate the clinical outcome of surgical treatment of degenerative disc disease (DDD) with the use of pedicular stabilization systems without fusion in 22 patients in the city of Aracaju/Sergipe. Methods: We used the visual analogic scale (VAS) and quality of life questionnaires (Oswestry and Roland Moris) preoperatively and 6 months after surgical treatment. According to the clinical symptoms and morpholophysiological changes found in the imaging exams, articular changes (facet degeneration), disc disease at adjacent levels, previous microdiscectomy, were determinant parameters for chose the use of implants that allow varying degrees of mobility. Results: Of the 22 patients studied, 16 were male and six female with mean age of $41.77 \pm 11.6$ years. A total of 43 levels were stabilized and the instrumentation from L4 to S1 was the most frequent segment (12) and the Dinamika ${ }^{\circledR}$ (GMReis - Brazil) plate for 2 levels was the most frequent used system (08), with fusion of the lower level (hybrid stabilization). There was significant improvement in symptoms when we observe a decrease of VAS preoperatively $(8,59 \pm 1,1)$ and 6 months postoperatively $(2,41 \pm 1,8)$ with improvement in quality of life scores in Oswestry and Roland Moris

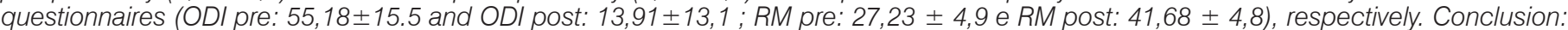
Although all patients included in the study had achieved a significant improvement both in quality of life and in reducing symptoms, further biomechanical studies should be performed in order to standardize an efficient approach to treat this pathology.
\end{abstract}

Keywords: Low back pain, Degenerative disc disease, Dynamic stabilization system.

\section{RESUMEN}

Objetivo: Evaluar el resultado clínico del tratamiento quirúrgico de la enfermedad del disco degenerativa (EDD) con la utilización de los sistemas de estabilización pedicular sin fusión en 22 enfermos en la ciudad de Aracajú/Sergipe.Métodos: Fueron utilizadas escalas visual analógica del dolor (EVA) y de calidad de vida (cuestionarios Oswestry e Rolland Morris) antes de la cirugía y seis meses después del tratamiento quirúrgico realizado. De acuerdo con las quejas clínicas y las alteraciones morfofisiológicas encontradas en los exámenes complementarios de imagen, grado de acometimiento articular (artrosis de las facetas), enfermedad discal en niveles adyacentes, microdiscectomías anteriores, se optó por la utilización de implantes que permiten grados de movilidad variables. Resultados: De los 22 enfermos que se incluyeron en el estudio, 16 eran del sexo masculino, y seis del sexo femenino, con el promedio de edad de 41,77 años, más o menos 11,6 años. Se estabilizó un total de 43 niveles, siendo la instrumentación de L4 a S1 la más frecuente (12) y el sistema más utilizado fue la placa Dinamika ${ }^{\circledR}$ (GMReis) para dos niveles (08), con fusión del nivel inferior (estabilización híbrida). Hubo mejoría importante de la sintomatología cuando observamos la disminución del EVA antes del procedimiento cirúrgico $(8,59$ más o menos 1,1) y seis meses después del procedimiento quirúrgico $(2,41$ más o menos 1,8), con mejora de la cualidad de vida segúnlas escalas de Oswestry e Rolland Morris (ODI antes: 55,18 más o menos 15,5 y ODI después: 13,91 más o menos 13,1; RM antes: 27,23 más o menos 4,9 y RM después: 41,68 más o menos 4,8), respectivamente. Conclusión: Aunque todos los enfermos incluidos en el estudio hayan obtenido una mejoría significativa tanto en la calidad de vida como en la disminución de la sintomatología, nuevos estudios biomecánicos deberán ser realizados para que se llegue a estandarizar un abordaje eficiente en el tratamiento de la patologia.

Descriptores: Dolor de espaldas, Discopatia degenerativa del disco; Estabilización dinámica

1. Membro da Sociedade Brasileira de Ortopedia eTraumatologia, Membro da Sociedade Brasileira de Coluna

Trabalho realizado pelo Grupo Coluna - CEOT (Centro de Especialidades em Ortopedia eTraumatologia)-Aracaju, SE, Brasil.

Correspondência: Grupo Coluna - CEOT (Centro de Especialidades em Ortopedia eTraumatologia).Rua Ananias Azevedo 505 - 13 de julho. CEP:49020-080 - Aracaju, SE, Brasil.

Recebido em 30/06/2010, aceito em 12/07/2011. 


\section{INTRODUÇÃO}

A lombalgia aguda faz parte da rotina diária dos atendimentos de emergência em ortopedia. Cerca de 2/3 da população adulta dos EUA sofrem de dor lombar sendo a segunda causa mais frequente de hospitalizações depois de infecções respiratórias altas¹. A doença discal degenerativa (DDD) é a patologia mais frequente nos ambulatórios de coluna, após o controle do quadro álgico nas unidades de urgência.

Em 2004 Adams e colaboradores descreveram os mecanismos envolvidos na gênese da dor lombar baixa, o comportamento do disco intervertebral ao suportar cargas, a distribuição dessas cargas no núcleo pulposo e a repercussão no ânulo fibroso, as lesão causadas por distúrbios biomecânicos e a cicatrização do disco intervertebral frente às injúrias sofridas ${ }^{2}$. As micro-fraturas encontradas em platôs vertebrais de cadáver levaram os autores a acreditar que forças compressivas repetidas (axiais) são os mecanismos mais frequentes de lesão discal ${ }^{2}$. A junção de compressão com a flexão está mais associada com as protrusões discais, sendo que os movimentos de flexão e extensão puros, parecem estar ligados com a funcionalidade inadequada da musculatura (abdominal e paravertebral) e das articulações facetarias ${ }^{3,4,5}$.

Características genéticas, idade, deficiências no transporte de metabólitos intradiscal, e má distribuição de peso (cargas axiais) no segmento lombar, podem levar a um enfraquecimento do ânulo fibroso e posterior ruptura com dano estrutural do disco intervertebral, que possui uma característica progressiva e irreversível. Sendo, talvez, a união dessas ocorrências a melhor definição de DDD6.

A definição de instabilidade segmentar, na coluna lombar (CL) requer uma análise quantitativa e qualitativa dos movimentos realizados ao longo dos 3 eixos, coronal, sagital e axial $(x, y, z)$. Essa avaliação pode ser dividida em estabilidade estática e dinâmica, sendo necessário o estudo criterioso da incapacidade da musculatura lombar e o SNC em regular o feedback com (devido aos possíveis danos teciduais, degradação das informações sensitivas e alterações de força muscular) a estabilidade fisiológica da coluna ${ }^{7}$.

Sengupta e cols. propuseram terminologia a fim de esclarecer a nomenclatura adequada a ser utilizada nos implantes que não imprimiriam fusão lombar (estabilização dinâmica, estabilização flexível), além de explicar o funcionamento das próteses acessíveis, naquele momento, orientavam para as peculiaridades de cada, a possibilidade de falência bem como a necessidade de estudos prospectivos randomizados para a prática de uma medicina baseada em evidências ${ }^{8}$.

Strempel e colaboradores realizaram uma comparação entre artrodese segmentar e um método de não fusão utilizando um sistema semi-rígido, que permite uma alta estabilidade em relação às forças de rotação, podendo assim ser utilizado em pacientes que possuem artrose facetaria, estenose vertebral sintomática e síndrome pós-laminectomia, obtendo melhora importante quando analisadas as reduções dos índices das escalas EVA e Oswenstry9.

$\mathrm{O}$ avanço de novas tecnologias e a maior compreensão biomecânica da coluna lombar trouxe propostas de tratamento cirúrgico menos invasivos, reduzindo as complicações decorrentes da artro-

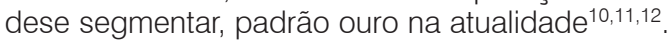

Vários dispositivos estão sendo comercializados com a finalidade de se obter um método que permita movimentos controlados da unidade funcional da coluna vertberal. $\mathrm{O}$ uso de parafusos pediculares sem a necessidade de fusão vem sendo "ofertados" na literatura atual com uma nova era de implantes que possibilitariam uma melhora ou aprimoramento no tratamento da DDD diminuindo os riscos cirúrgicos em comparação com a artrodese (tais como sangramento, tempo cirúrgico, risco de lesões neurológicas, sobrecarga nível adjacente) $)^{1,8,11}$.

O objetivo do nosso estudo é avaliar o resultado clínico do uso de três diferentes tipos de implantes para o tratamento da DDD, que permitem graus variáveis de movimento, com critérios, definidos no pré-operatório, de inclusão para uso de sistemas dinâmicos (Dynesys $^{\circledR}$ ), Semi-rígidos (Cosmic ${ }^{\circledR}$ (Ulrich) e Placa Dinamika ${ }^{\circledR}$ (GMReis) para um nível) e Híbrido (Placa Dinamika ${ }^{\circledR}$ (GMReis) para dois níveis).

\section{MATERIAIS E MÉTODOS}

Foram estudados 22 pacientes no período de 2008 a 2010 submetidos a tratamento cirúrgico com métodos de não-fusão ou estabilização híbrida com três tipos diferentes de implantes (Placa Dinamika ${ }^{\circledR}$ (GMReis), Dynesis ${ }^{\circledR}$ (Zimmer) e Cosmic ${ }^{\circledR}$ (Ulrich)), a depender dos critérios de inclusão para cada implante, sendo avaliados o sexo, a média de idade, o número de níveis, os mais afetados, as complicações e utilizadas as escalas de dor EVA, Oswestry (ODI) e Rolland Moris (RM), validadas para língua portuguesa (Brasil), como método de avaliação da melhora sintomatológica e qualidade de vida dos pacientes, no pré-operatório e 6 meses após a cirurgia.

Por se tratarem de técnicas inovadoras que cujos estudos variam em grau de recomendação e força de evidência, os critérios de inclusão para cada sistema foi baseado nas publicações atuais sobre o perfil de movimento permitido por cada sistema e o debate direto com os fabricantes, engenheiros e médicos responsáveis pelo desenvolvimento dos materiais, em congressos/cursos nacionais e internacionais.

Afim de padronizar a inclusão dos pacientes para os diferentes tipos de implantes, os pacientes foram subdivididos em 4 classes:

A primeira foi constituída de pacientes que não tinham sido submetidos a cirurgias de coluna prévias, com dor lombar mecânica sem sinais de sobrecarga facetária/artrose facetária (RX ou TC), sem síndromes compressivas radiculares (parestesias e dor irradiada para MMII), sem necessidade de laminectomiadescompressiva e com instabilidade no exame radiográfico dinâmico $(\mathrm{RX})$, foram submetidos a estabilização com dispositivo dinâmico Dynesis $^{\circledR}$ (Zimmer)

A segunda por pacientes que possuíam hérnias de disco sintomáticas (radiculopatias, alodínias e parestesias em MMIIs) com necessidades de descompressão, associadas ou não a dor lombar mecânica, sem sinais de artrose facetária, podendo ou não haver instabilidade ao rx dinâmico e acometimento de apenas um segmento utilizamos o sistema semi-rígido (Placa Dinamika ${ }^{\circledR}$ (GMReis) para um nível.

Os pacientes com discopatia lombar baixa (L4/L5/S1), espondilolistese no nível inferior associada à doença discal adjacente do nível superior, necessidade de correção de deformidades (diminuição do espaço intersomático e espondilolistese) e necessidade de laminectomia ampla, utilizamos fixação híbrida (Placa Dinamika ${ }^{\circledR}$ (GMReis) dois níveis) com artrodese intersomático e posterolateral no nível inferior, esses pacientes compunham a terceira classe.

A quarta classe foi constituída de pacientes com artrose facetária, doença discal degenerativa em até três níveis, microdiscectomias prévias com dor lombar mecânica (síndrome pós-laminectomia), utilizamos o implante semi-rígido (Cosmic ${ }^{\circledR}$ (Ulrich)), pois o sistema não permite movimentos rotacionais e lateralizações.

Quanto maior as alterações degenerativas no complexo motor triarticular, observadas nos exames pré-operatório, maior a necessidade de se restringir os movimentos acoplados, sendo assim pacientes que possuíam alterações facetárias foram incluídos em sistemas que limitam movimento nos 3 eixos. No entanto, pacientes com doença discal degenerativa sem história de cirurgias prévias e poucas alterações anatômicas evidenciadas no acompanhamento clínico foram incluídos em sistemas que permitem maior mobilidade.

Todos os pacientes que possuíam artrose facetária importante e necessidade de descompressão foram submetidos a acesso mediano posterior com dissecção subperiosteal e laminectomia tradicional (Ampla, Hemilaminctomia ou Hemi-hemi).

Pacientes jovens e sem sinais de artrose facetária foram submetidos ao acesso de Wiltse e caso necessário descompressão, e/ou artrodese no nível inferior, foi realizada por via transforaminal (TLIF).

Todos os pacientes da amostra assinaram termo de consentimento livre e esclarecido e a pesquisa teve aprovação junto ao comitê de ética da Clinica de Especialidades em Ortopedia e Traumatologia de Sergipe (CEOT).

A análise estatística foi realizada pelo programa estatístico SPSS ${ }^{\circledR}$ versão 11.0 para Windows. Os dados foram apresentados em média e desvio padrão. 


\section{RESULTADOS}

Dos 22 pacientes inseridos no estudo 16 (72,7\%) eram do sexo masculino e $6(27,3 \%)$ do sexo feminino, com media de idade de $41,77 \pm 11,6$ anos.

Cinco pacientes apresentavam laminectomia prévia para tratamento de hérnia de disco com radiculopatia e retorno da sintomatologia.

Foram estabilizados um total de 43 níveis sendo a instrumentação de L4 a S1 a mais frequente (12) seguida de L5/S1(06) e L3 à S1(05).

Dos sistemas utilizados a placa Dinâmica ${ }^{\circledR}$ (GMReis) para dois níveis foi a mais utilizada (08), seguidos em ordem decrescente dos seguintes sistemas: Cosmic ${ }^{\circledR}$ (Ulrich) três níveis (04), Cosmic ${ }^{\circledR}$ (Ulrich) um nível (03) e Dinamika ${ }^{\circledR}$ (GMReis) um nível (03), Dynesis ${ }^{\circledR}$ (Zimmer) dois níveis (02), Cosmic ${ }^{\circledR}$ (Ulrich) dois níveis (01) e Dynesis ${ }^{\circledR}$ (Zimmer) três níveis (01).

Houve melhora significativa da sintomatologia quando observamos a diminuição da EVA no pré-operatório $(8,59 \pm 1,1)$ e seis meses no pós-operatório $(2,41 \pm 1,8)$, com melhora significativa na qualidade de vida nas escalas de Oswestry e Roland Moris ( ODIpré: 55,18 \pm 15,5 e ODlpós: 13,91 \pm 13,1 ; RMpré: 27,23 \pm 4,9 e RMpós: 41,68 $\pm 4,8)$, respectivamente.

Dois pacientes apresentaram parestesias residuais em membros inferiores com resolução espontânea após quatro meses de pós-operatório.

Um paciente apresentou déficit motor da raiz de S1 dir., apesar de estudos de imagem (RX, TC e RNM) evidenciar o bom posicionamento dos implantes, foi realizada revisão com ampliação da laminectomia e identificar integridade da raiz acometida.

Houve um episódio ruptura do segmento móvel da placa Dinamika $^{\circledR}$ (GMReis) (2 níveis) sem sintomatologia e com reação óssea importante ao redor dessa falha obtendo uma artrodese póstero-lateral.

\section{DISCUSSÃO}

Na literatura atual, observamos variadas indicações para o uso dos dispositivos pediculares sem fusão, porém sem critérios fundamentados para a utilização de cada sistema isoladamente, a depender das alterações clínicas e de imagem perceptíveis ao cirurgião.

Em nosso meio, Di Ferreira ${ }^{1}$ em um estudo prospectivo observacional de 39 pacientes submetidos a tratamento cirúrgico com estabilização pedicular dinâmica demonstrou uma melhora significativa na dor lombar e qualidade de vida com redução nos índices EVA e Oswenstry. Vale ressaltar que os autores não incluiram pacientes com artrose facetaria, utilizaram um acesso cirúrgico que causa menos lesões às estruturas estabilizadoras posteriores (músculos, articulações e ligamentos) e que, quando havia a necessidade de descompressão radicular, foi realizada microdiscectomia por minincisão ${ }^{1}$. Estas indicações talvez possam sugerir, que dispositivos muito móveis (que permitem um movimento maior nos três eixos) necessitam de uma maior participação dos elementos anatômicos, e da sua integridade, para um funcionamento biomecânico mais adequado.

\section{REFERÊNCIAS}

1. Di Ferreira RB, Daher $S$, Cardoso AP, Júnior WEP, Júnior ZAS, Tristão NA. Tratamento cirúrgico da doença degenerativa discal com estabilização dinâmica.Coluna. 2008;7(3)217-222.

2. Adams MA. Biomechanics of back pain. Acupuncture in medicine 2004; 22(4):178-188.

3. Adams MA, Roughley PJ. What is Intervertebral Disc Degeneration, and What Causes It? Spine.2006; 31(18): 2151-2161.

4. Deyo RA, Weinstein J. Low Back Pain. N Engl J Med. 2001; 344(5): 263-270.

5. Oliveira VM, Puertas ED, Alves MS, Chagas JCM, Oliveira CEAS, Santos FPE. Estudo das terminações nervosas dos discosintervertebrais da coluna lombar de humanos. Rev Bras Ortop. 2005; 37(5)195-200.

6. Jensen TE ,Kjaer P, Korsholm L,Bendix O, Sorensen JS ,Manniche C,Leboeuf-Y. Predictors of new vertebral endplate signal (modic)changes in the general population. Eur Spine J.2010: (19):129-135.

7. Reeves NP, Narendra KS, Cholewicki J. Spine stability: the six blind men and the elephant.ClinBiomech (Bristol, Avon). 2007; 22(3): 266-274.
As articulações facetárias sobrecarregadas e com sinais de desgaste (prematuro ou não) são importante fonte de origem de dor e processo inflamatório crônico. Portanto, o controle adequado das forças de rotação no segmento lombar pode diminuir o contato articular patológico ou de estresse. O uso de dispositivos que controlem movimentos rotacionais, como Cosmic ${ }^{\circledR}$ (Ulrich), deve ser avaliado pelo cirurgião em pacientes que estejam com acometimento dessas articulações em exames de imagens (Rx+TC) e suspeita clínica de síndrome facetária. Talvez por essa característica intrínseca desse implante, os desenvolvedores o contra-indicam para correção de deformidades (escolioses e quando se deseja corrigir uma espondilolistese), sendo esse dispositivo indicado para patologias que possam interferir com as instabilidades rotacionais (síndromes pós-laminectomias, espondilolisteses de baixo grau) ${ }^{9}$.

Por outro lado, pacientes que possuem uma diminuição importante do espaço intersomático no nível inferior (L5/S1) com artrose facetária importante e consequente redução da amplitude foraminal (estenose do recesso lateral) com sinais clínicos e laboratoriais de início de doença no nível adjacente (sinais de degeneração discal com lesão do ânulo fibroso, protrusão discal ) podem necessitar de uma melhor correção axial e sagital. Tal correção poderá ser mais bem alcançada com a artrodese intersomática no nível inferior. Sistemas como Aladyn ${ }^{\circledR}$, Isobar TTL ${ }^{\circledR}$ (Scient'X) e em nosso meio Placa Dinamika $^{\circledR}$ (GMReis) têm a característica de permitir o acoplamento de duas técnicas, fusão e não fusão em um só sistema. Esta propriedade permite alcançar um tratamento mais harmonioso com as diversas alterações, que muitas vezes encontram-se sobrepostas no tratamento da DDD multisegmentar.

A necessidade de uma maior compreensão das alterações morfológicas/funcionais e sua associação com as sintomatologias apresentadas nos pacientes portadores de DDD poderá determinar a quantidade de movimento necessária para uma estabilização posterior sem fusão, aumentando o sinergismo biomecânico ente os implantes e a CL. Apesar da ausência de significância estatística $(p>0,05)$ nas variáveis avaliadas em nosso estudo, talvez pela pequena amostra e curto período de segmento, os pacientes obtiveram uma melhora clínica importante. Em especial quando observamos as pontuações obtidas nas escalas de avaliação da qualidade de vida, denotando que uma avaliação mais apurada das mudanças estruturais e biomecânicas dos segmentos acometidos no pré-operatório pode sugerir a utilização de uma órtese com maior ou menor mobilidade individual, a fim de permitir a quantidade necessária de movimento indolor.

\section{CONCLUSÃO}

Os métodos de estabilização da coluna lombar por meio de parafusos pediculares, sem a intenção de se obter a fusão, parecem ser uma forma promissora de tratamento das doenças discais degenerativas, com melhora importante nos escores de qualidade de vida e de dor. Sendo necessário, porém, uma melhor determinação pré-operatória da quantidade de movimento que se deseja obter após a correção cirúrgica, para a escolha de um implante mais adaptado para a realidade clínica de cada paciente.
8. SenguptaDK.Dynamic Stabilization Devices in the treatment of Low Back Pain. Coluna.2005; 4(1): 31-41.

9. Strempel Av, Stoss C, Moosmann D, Martin A. Non-fusion stabilization of the lumbar spine in the case ofdegenerative diseases with a dynamic pedicle screw rod. Coluna. 2006; 5(1):13-18.

10. SchulteTL, HurschlerC, Haversath M, Liljenqvist U, Bullmann V, FillerTJ et al. The effect of dynamic, semi-rigid implants on the range of motion of lumbar motion segments after decompression. Eur Spine J.2008:17:1057-1065

11. Lee S, Park S, Jahng T, Chung C, Kim H. Clinical Experience of the DynamicStabilization System for the DegenerativeSpine Disease. J Korean Neurosurg Soc. 2008; $43: 221-226$.

12. Monmeneu MB, García VB, Baeza FR, Sáez D. Sistema de neutralización dinámica en la columna lumbar. SISTEMA DYNESYS. Experiencia en 94 casos. Neurocirugía 2005; 16:499-506. 